Call for Correspondence JNCCN is committed to providing a forum to enhance collaboration between academic medicine and the community physician. We welcome comments about the NCCN Clinical Practice Guidelines in Oncology (NCCN Guidelines), articles published in the journal, or any other topic relating to cancer prevention, detection, treatment, supportive care, or survivorship.

Please send correspondence to JNCCN.edmgr.com or to JNCCN@nccn.org.

Letters should be no more than 400 words, with no more than 5 references if included. Please include the full names, degrees, and affiliations of all letter authors and a phone number or e-mail address for contact.

Letters are considered for publication as space allows. NCCN reserves the right not to publish correspondence for any reason it deems appropriate. All letters are subject to editing and/or abridgment.

\title{
Letter to the Editor: Significance of Clonal Hematopoiesis of Indeterminate Potential
}

Re: Rachel L. Mitchell, Cory Kosche, Kelly Burgess, et al. Misdiagnosis of LiFraumeni Syndrome in a Patient With Clonal Hematopoiesis and a Somatic TP53 Mutation. J Natl Compr Canc Netw 2018;16(5):461-466.

Mitchell et al ${ }^{1}$ nicely demonstrated that their patient, diagnosed in 2014 as a Li-Fraumeni family member harboring a germline TP53 deleterious mutation, was misdiagnosed, and they concluded that the patient instead had "clonal hematopoiesis $(\mathrm{CH})$ at the time of genetic testing," not a germline TP53 mutation.

However, as they noted, although $2 \%$ to $20 \%$ is an accepted range for $\mathrm{CH}$ or $\mathrm{CH}$ of indeterminate potential (CHIP), their patient's cell-free (cf) DNA mutation allelic frequency (MAF) was $50 \% \cdot{ }^{1-3}$ With such high MAFs, I believe that screening for precancer conditions or occult malignancies should be considered when, as the authors showed, the known malignancy does not harbor the same TP53 mutation and normal tissue demonstrates no TP53 mutation.

Multiple studies have now shown that CHIPs are fairly common and the rate of transformation to malignancy is roughly $0.5 \%$ to $1 \%$ per year. ${ }^{3}$ However, a relatively high MAF at the time of CHIP diagnosis predicts a higher likelihood of transformation. ${ }^{2-4}$ For example, in one of the landmark CHIP studies, a subject with a 24\% TP53 MAF developed acute leukemia 34 months later. At that point, the patient showed an 84\% TP53 MAF. 4

Next-generation sequencing of cfDNA either to identify a germline mutation or as a "liquid biopsy" to identify actionable abnormalities for targeted therapies has become increasingly common. Although a low TP53 DNA MAF suggests only a very small likelihood of transformation, I believe physicians should consider screening patients with a high cf TP53 DNA MAF (despite whether they harbor a germline TP53 deleterious mutation [Li-Fraumeni]) for a premalignant condition or an occult malignancy associated with somatic mutations in TP53, provided their known cancer does not demonstrate that same mutation, as an explanation for the high MAF.

I agree with the authors that their patient was not a Li-Fraumeni family member, but disagree with their conclusion that excluding Li-Fraumeni syndrome was sufficient evidence to conclude that the diagnosis should instead have been $\mathrm{CH}$, without first evaluating for a precancer condition or an occult malignancy.

Steven Sorscher, MD

Wake Forest School of Medicine

Winston-Salem, NC

Email: ssorsche@wakehealth.edu doi: 10.6004/jnccn.2018.7075

\section{References}

1. Mitchell RL, Kosche $C$, Burgess $K$, et al. Misdiagnosis of Li-Fraumeni syndrome in a patient with clonal hematopoiesis and a somatic TP53 mutation. J Natl Compr Canc Netw 2018;16:461-466

2. Steensma DP, Bejar R, Jaiswal S, et al. Clonal hematopoiesis of indeterminate potential and its distinction from myelodysplastic syndromes. Blood 2015;126:9-16.

3. Jaiswal S, Fontanillas P, Flannick J, et al. Age related clonal hematopoiesis associated with adverse outcomes. N Engl J Med 2014;371:24882498.

4. Genovese G, Kahler AK, Handsaker ER, et al. Clonal hematopoiesis and blood-cancer risk inferred from blood DNA sequence. N Engl J Med 2014;371:2477-2487. 


\section{Authors' Reply to Letter to the Editor: Significance of Clonal Hematopoiesis of Indeterminate Potential}

\begin{abstract}
Authors' Reply to Steven Sorscher's Letter to the Editor re: Rachel L. Mitchell, Cory Kosche, Kelly Burgess, et al. Misdiagnosis of Li-Fraumeni Syndrome in a Patient With Clonal Hematopoiesis and a Somatic TP53 Mutation. J Natl Compr Canc Netw 2018;16(5):461-466.
\end{abstract}

We appreciate the author's questions and comments on our article. ${ }^{1}$ We agree that, in general, ruling out a germline mutation is not sufficient to attribute a molecular abnormality found on germline testing to clonal hematopoiesis $(\mathrm{CH})$. However, in our case, it was a logical conclusion because the particular TP53 mutation was found in the leukemic bone marrow of our patient. TP53 mutations in blood cells are commonly associated with $\mathrm{CH}$, and $\mathrm{CH}$ may result in leukemia. Unfortunately, this finding-and the confirmation that the TP53 mutation in her leukemic marrow was different from the TP53 mutation in her ovarian cancer-was discovered after the patient's death in our attempt to clarify a cancer risk for her relatives given her previous diagnosis of $\mathrm{Li}$-Fraumeni syndrome.

We argue that in her case, a search for an occult malignancy based on the identified molecular abnormality would not have been warranted because she had a known advanced ovarian cancer at the time of germline testing and thus, was followed closely with body scan to assess response to therapy.

Dr. Sorscher has brought up an important question on how to manage potentially significant somatic mutations incidentally found on germline testing, especially in a per- son without prior history of cancer. Although there is an expanding body of literature on managing incidentally found germline mutations on tumor profiling, ${ }^{2,3}$ there is a paucity of data on managing somatic mutations incidentally discovered on germline testing for cancer predisposition. This may be due to multiple possible causes of this finding, including mosaicism, $\mathrm{CH}$, malignancy, and senescence. Currently, no guidelines are available, and each case is managed based on individual clinical circumstances. A workup for an occult malignancy may not be covered in the absence of apparent malignancy. If $\mathrm{CH}$ is suspected, these patients may be followed up by a hematologist.

We suggest that testing laboratories consistently report genetic mutations even if found at a lower allelic fraction; these individuals may then be studied prospectively to develop algorithms on how to approach them. For cases in which an allelic fraction is close to $50 \%$, the ability to accurately discriminate somatic and germline mutations remains critical. Advances in bioinformatics may help solve this problem, ${ }^{4}$ as the detective work we and other authors ${ }^{5}$ had to do in such cases may not be always possible.

Lydia Usha, MD

Rush University Medical Center Chicago, IL Email:lydia_usha@rush.edu

Kelly Burgess, MD, CGC Rush University Medical Center Oleksandra Klapko, BS Rush University Medical Center Lela Buckingham, MD Rush University Medical Center doi: 10.6004/jnccn.2018.7076

\section{References}

1. Mitchell RL, Kosche C, Burgess $\mathrm{K}$, et al. Misdiagnosis of Li-Fraumeni syndrome in a patient with clonal hematopoiesis and a somatic TP53 mutation. J Natl Compr Canc Netw 2018;16:461-466.

2. Ngeow J, Eng C. Precision medicine in heritable cancer: when somatic tumour testing and germline mutations meet. NPJ Genom Med 2016;1:15006.

3. Catenacci DV, Amico AL, Nielsen SM, et al. Tumor genome analysis includes germline genome: are we ready for surprises? Int J Cancer 2015;136:1559-1567.

4. $\mathrm{Hu}$ Y, Alden RS, Odegaard JI, et al Discrimination of germline EGFR T790M mutations in plasma cell-free DNA allows study of prevalence across 31,414 cancer patients. Clin Cancer Res 2017;23:73517359

5. Sorscher S, Desnoyers R, Ouyang K, et al. Li-Fraumeni versus pseudo-Li-Fraumeni syndrome: key insights for interpreting next-generation sequencing reports in patients with suspected cancer predisposition syndromes. Oncologist 2017;22:1084-1085. 